\title{
Clinical Neuroscience-Towards a Better Understanding of Non-Conscious versus Conscious Processes Involved in Impulsive Aggressive Behaviours and Pornography Viewership
}

\author{
Sajeev Kunaharan1, Peter Walla1,2* \\ ${ }^{1}$ Functional Neuroimaging Lab, Centre for Translational Neuroscience and Mental Health Research, School of \\ Psychology, University of Newcastle, Newcastle, Australia \\ ${ }^{2}$ Department of Psychology, Webster Private University Vienna, Palais Wenkheim, Vienna, Austria \\ Email: ${ }^{*}$ peter.walla@newcastle.edu.au
}

Received 9 October 2014; revised 2 November 2014; accepted 17 November 2014

Copyright (C) 2014 by authors and Scientific Research Publishing Inc.

This work is licensed under the Creative Commons Attribution International License (CC BY).

http://creativecommons.org/licenses/by/4.0/

(c) (i) Open Access

\begin{abstract}
Assuming that the human mind indeed consists of a non-conscious and a conscious part it makes sense to believe that consciousness at times may struggle to get access to non-conscious content, which seems rather logical. At the same time most of us are aware that affective processing underlying our emotions happens non-consciously due to limbic activity that is mostly sub-cortical. Thus, any explicit response to a question about one's state of affect is inevitably prone to be inaccurate if not wrong. Therefore, any therapy, biological and/or psychological that is based on explicit responses is potentially misleading. With this opinion article we aim to generate awareness about potential discrepancies between self-reported versus objectively measured emotion-related states. There is more to emotion than just subjective feeling and we should start taking nonconscious emotion-related processes into account.
\end{abstract}

\section{Keywords}

Non-Conscious Affective Processing, Implicit versus Explicit Responses, Electroencephalography (EEG), Startle Reflex Modulation (SRM), Emotion

\footnotetext{
${ }^{*}$ Corresponding author.

How to cite this paper: Kunaharan, S., \& Walla, P. (2014). Clinical Neuroscience-Towards a Better Understanding of NonConscious versus Conscious Processes Involved in Impulsive Aggressive Behaviours and Pornography Viewership. Psychology, 5, 1963-1966. http://dx.doi.org/10.4236/psych.2014.518199
} 


\section{Introduction}

Little has been made mention over the years of diagnostic practices currently employed in the behavioural sciences and the apparent drawbacks they may have. Current protocols in the diagnosis and subsequent treatment of behavioural disorders by clinicians largely stem from structured interviews whereby the clinician will follow guidelines for diagnosis and classification outlined in the Diagnostic and Statistical Manual IV (DSM IV-TR) (APA, 2000) and/or International Classification of Diseases 10 (ICD 10) (WHO, 2004). This process usually involves an interview session followed by questionnaires, which are filled out by the patient aiming to aid the clinician in diagnosis. Objective evaluation is usually only used if the clinician believes that the behavioural problem is a symptom of an underlying medical issue in which case the patient will be referred to a medical practitioner. Researchers in recent years have recognised that this current framework supplied by the DSM-IV and the ICD-10 has serious shortcomings regarding its validity due to several factors in particular: comorbidities among certain diagnoses and an over-specification of specific categories. Additionally, many categories were created, whilst techniques to measure objective physiological processes such as Electroencephalography (EEG) and Startle Reflex Modulation (SRM) (both discussed later) were still in their infancy and hence these measures lacked a biological basis in their diagnostic criteria (Insel \& Cuthbert, 2009; Robbins et al., 2012). As a result, it seems that the following question should be asked: why shouldn't objective measures also be used in the interpretation and diagnosis of the human psyche when they are ubiquitously used to aid the diagnosis and treatment of most other medical conditions? Given this question, we need to formulate methods in order to firstly, ascertain whether physiological measures - which are more adept at revealing our non-conscious thoughts and feelings - are a more accurate representation of how a person feels, and secondly, whether this information once gathered will be useful in aiding clinicians in the diagnosis and treatment of behavioural disorders.

\section{What Measures Can We Use?}

Human behaviour can be thought of as a combination of processes involving both cognitive and affective components (Walla et al., 2014). Investigations using self-reporting techniques only allow us to measure the cognitive aspect of human behaviour whilst the lesser understood affective processing which is largely non-conscious is not considered (Walla \& Panksepp, 2013). Self-reporting provides the clinician with "conscious" or "explicit" feedback from the patient, which is assumed to be an accurate representation of their innermost attitudes and feelings. Till now, this has been the method most widely used to provide the clinician with the most effective diagnosis and treatment options within the behavioural sciences. If these inaccurate self-reporting measures are being used to determine treatment options, then these treatment options aren't able to uncover the underlying behavioural issue of these individuals. The consequences of solely utilising the aforementioned methods to understand one aspect of this behavioural dichotomy may be deleterious. The lack of recognition of these underlying behavioural issues and consequently the providing of ineffective treatment may not adequately modify problem behaviours and may show an increase in behaviours which can lead to negative consequences (e.g. violent crime, sexual assault etc.) in the society that they live in.

As there are consequences in the use of self-report questionnaires to measure "non-conscious" or "implicit" behaviours, certain methods employed in neuroscience will be suggested in order to add weight to its use as a reliable measure of implicit or non-conscious behaviours. We acknowledge however that not all neuroscientific methods may be suitable for this purpose. Commonly used techniques to study brain processing such as fMRI (Functional Magnetic Resonance Imaging) for example, although widely used in the neurosciences is quite costly and limited in its temporal resolution and this delay in data acquisition doesn't allow it to be suitable for capturing fast and short implicit neural activity. Studies however, incorporating the use of Electroencephalography (EEG) have recorded participant neural activity reflecting implicit attitudes in real-time (Bosshard \& Walla, 2013). This is more than adequate in recording neural impulses, which occur below conscious awareness and thus may be the most reliable instrument we can use to measure implicit neural activity. Another measure which has been long used to measure non-conscious processing is Startle Reflex Modulation (SRM). SRM has been used in several studies to selectively measure the valence of neural processing by measuring the intensity of eye blinks when presented with a startle probe (Bradeley et al., Grahl et al., 2012; Mavratzakis et al., 2013). The non-conscious behavioural effect occurs as people are usually more startled and hence, deliver a more intense blink when viewing something they innately find disruptive or disturbing and not so much when they view something they find pleasurable. 


\section{Targeted Research Groups}

Due to the significant role our non-conscious plays in our behaviours, it is of course redundant to mention that several behavioural disorders could make use of further knowledge and understanding of its components. However, a closer look at specific questions relating to conscious vs non-conscious mechanisms is important as it allows for the focus of specific behavioural characteristics to be explored. This allows for more streamlined research in this area of study, driving the search more specifically to particular groups.

Prior studies have aimed to investigate whether conscious exposure to violent or sexual behaviour (be it via movies, television, video games, magazines, internet etc.) has any effect on an individual's behaviour or thought processes with regards to aberrant sexual and/or aggressive behaviours (Engelhardt et al., 2011; Anderson et al., 2010; Krahé \& Möller, 2011; Willoughby et al., 2014; Svedin \& Priebe, 2011). Research in this area is incredibly important as it is taking place at a time where society is exploding with continual technological advances, in particular increased access to a myriad of online content. This in turn, has allowed an ever increasing availability of sexually explicit and violent material available to anyone who wishes to access it. An observable issue with many of the aforementioned studies is the reliance of self-report measures in acquiring data on individual attitudes. It is necessary to take a close look at the implications of the inherent biases encountered during selfreporting. In particular, as this research is predominantly focusing on matters involving aggression and sex, the taboo nature of such topics gives rise to an individual subjectively tailoring his/her responses to the more socially/and or culturally appropriate response. This leaves us with results that don't accurately reflect the demographics' tendencies.

Further to this, aggression has historically been seen as a homogeneous construct where despite its multifaceted nature, researchers have rarely differentiated between its differing forms. Venturing deeper to look at aggression as a behavioural characteristic, it is often a component of clinical disorders that is associated with abnormal brain functioning (Siegel \& Victoroff, 2009). A sub-class of aggression known as impulsive aggression should be focused on as it may be more relevant than premeditated aggression (the other sub-class of aggression) when studying individuals who view pornographic material as many pornography users often compulsively view this material and impulsive and compulsive behaviours have been shown to be somewhat related (Robbins et al., 2012; Robbins et al., 2012; Fineberg et al., 2014). As mentioned previously, it makes more sense to streamline research into particular areas such as this as it allows more useful and therefore usable data to be collected.

\section{Take Home Message}

The main take home message of this opinion piece is to drive home the notion that there is a lot more processing going on in the non-conscious mind than we care to think about and the use of surveys doesn't allow us to take this information into consideration. Self-reported data or face to face interviews largely only give us the conscious or explicit aspect of an individual's behaviour and doesn't at all reflect the individual's non-conscious or implicit viewpoint. The main focal point of this research looks at impulsive aggressive individuals and people who view pornography. Both behaviours (aggression and sex) targeted by these groups are evolutionally incredibly important and are largely driven by instinct with little or no higher cognitive resources involved. As it is so, it makes more sense to measure and categorise these behaviours using implicit physiological measures rather than what is currently being used, which allows responses to be deliberate evaluations made cognitively after careful reasoning and evaluation of consequences.

\section{Conclusion}

It may be necessary to educate further and inform clinicians with what needs to be growing research into how they may better categorise, diagnose and finally treat their patients' behavioural disorders. By only targeting one aspect of human behaviour they are not seeing the full picture of exactly what a behavioural characteristic entails and are therefore not in the best position to understand and treat them. The more we know about the non-conscious processes that drive human behaviour, the more clinicians may be able to better form treatment options for certain behavioural problems. Methods employed in the neurosciences, most specifically EEG and SRM may serve us best as instruments used to ascertain these non-conscious processes whereby physiological markers specific to certain behaviours may be present, which can be used to aid in diagnosis. This opinion ar- 
ticle hopes to provide insight in order to possibly advance existing knowledge to clinicians and also provide a guideline from which future amendments may be made by the American Psychological Association and The World Health Organisation to their respective diagnostic manuals.

\section{References}

American Psychiatric Association (Ed.) (2000). Diagnostic Criteria from dsM-iV-tr. Arlington: American Psychiatric Pub Incorporated.

Anderson, C., Shibuya, A., Ihori, N., Swing, E., Bushman, B., Sakamoto, A., \& Saleem, M. (2010). Violent Video Game Effects on Aggression, Empathy, and Prosocial Behavior in Eastern and Western Countries: A Meta-Analytic Review. Psychological Bulletin, 136, 151-173. http://dx.doi.org/10.1037/a0018251

Bosshard, S., \& Walla, P. (2013). Objective Measures within Consumer Neuroscience. Frontiers in Human Neuroscience. Conference Abstract: ACNS-2013 Australasian Cognitive Neuroscience Society Conference.

Engelhardt, C. R., Bartholow, B. D., Kerr, G. T., \& Bushman, B. J. (2011). This Is Your Brain on Violent Video Games: Neural Desensitization to Violence Predicts Increased Aggression Following Violent Video Game Exposure. Journal of Experimental Social Psychology, 47, 1033-1036. http://dx.doi.org/10.1016/j.jesp.2011.03.027

Fineberg, N. A., Chamberlain, S. R., Goudriaan, A. E., Stein, D. J., Vanderschuren, L. J. M. J., Gillan, C. M., \& Potenza, M. N. (2014). New Developments in Human Neurocognition: Clinical, Genetic, and Brain Imaging Correlates of Impulsivity and Compulsivity. CNS Spectrums, 19, 69-89. http://dx.doi.org/10.1017/S1092852913000801

Grahl, A., Greiner, U., \& Walla, P. (2012). Bottle-Shape Elicits Gender-Specific Emotion: A Startle Reflex Modulation Study. Psychology, 3, 548-554. http://dx.doi.org/10.4236/psych.2012.37081

Insel, T. R., \& Cuthbert, B. N. (2009). Endophenotypes: Bridging Genomic Complexity and Disorder Heterogeneity. Biological Psychiatry, 66, 988-989. http://dx.doi.org/10.1016/j.biopsych.2009.10.008

Krahé, B., \& Möller, I. (2011). Links between Self-Reported Media Violence Exposure and Teacher Ratings of Aggression and Prosocial Behavior among German Adolescents. Journal of Adolescence, 34, 279-287. http://dx.doi.org/10.1016/j.adolescence.2010.05.003

Mavratzakis, A., Molloy, E., \& Walla, P. (2013). Modulation of the Startle Reflex during Brief and Sustained Exposure to Emotional Pictures. Psychology, 4, 389-395. http://dx.doi.org/10.4236/psych.2013.44056

Robbins, T. W., Curran, H. V., \& de Wit, H. (2012). Special Issue on Impulsivity and Compulsivity. Psychopharmacology, 219, 251-252. http://dx.doi.org/10.1007/s00213-011-2584-X

Robbins, T. W., Gillan, C. M., Smith, D. G., de Wit, S., \& Ersche, K. D. (2012). Neurocognitive Endophenotypes of Impulsivity and Compulsivity: Towards Dimensional Psychiatry. Trends in Cognitive Sciences, 16, 81-91. http://dx.doi.org/10.1016/j.tics.2011.11.009

Siegel, A., \& Victoroff, J. (2009). Understanding Human Aggression: New Insights from Neuroscience. International Journal of Law and Psychiatry, 32, 209-215. http://dx.doi.org/10.1016/j.ijlp.2009.06.001

Svedin, C. G., Åkerman, I., \& Priebe, G. (2011). Frequent Users of Pornography. A Population Based Epidemiological Study of Swedish Male Adolescents. Journal of Adolescence, 34, 779-788. http://dx.doi.org/10.1016/j.adolescence.2010.04.010

Walla, P., \& Panksepp, J. (2013). Neuroimaging Helps to Clarify Brain Affective Processing without Necessarily Clarifying Emotions. In K. N. Fountas (Ed.), Novel Frontiers of Advanced Neuroimaging. Winchester: InTech.

Walla, P., Koller, M., \& Meier, J. L. (2014). Consumer Neuroscience to Inform Consumers_Physiological Methods to Identify Attitude Formation Related to Over-Consumption and Environmental Damage. Frontiers in Human Neuroscience, 8, 304. http://dx.doi.org/10.3389/fnhum.2014.00304

Willoughby, B. J., Carroll, J. S., Nelson, L. J., \& Padilla-Walker, L. M. (2014). Associations between Relational Sexual Behaviour, Pornography Use, and Pornography Acceptance among US College Students. Culture, Health \& Sexuality, 16, 118. http://dx.doi.org/10.1080/13691058.2014.927075

World Health Organization (2004). ICD-10: International Statistical Classification of Diseases and Related Health Problems. Geneva: WHO. 
Scientific Research Publishing (SCIRP) is one of the largest Open Access journal publishers. It is currently publishing more than 200 open access, online, peer-reviewed journals covering a wide range of academic disciplines. SCIRP serves the worldwide academic communities and contributes to the progress and application of science with its publication.

Other selected journals from SCIRP are listed as below. Submit your manuscript to us via either submit@scirp.org or Online Submission Portal.
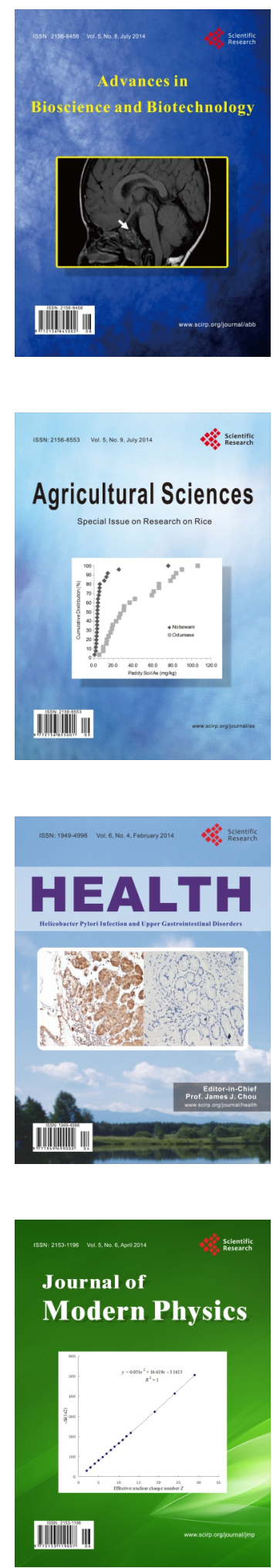
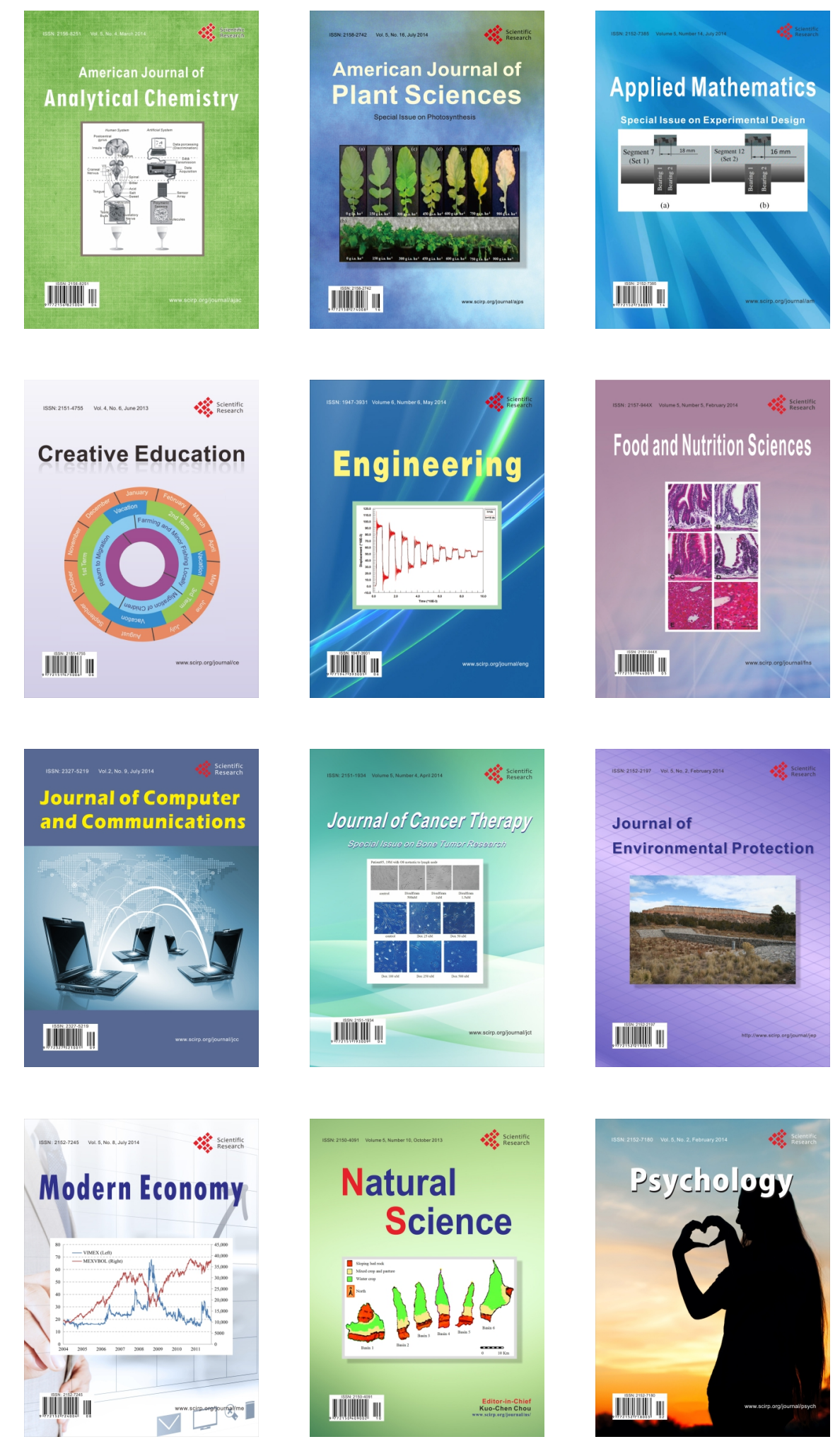